\title{
Reducing Power and Increasing Accuracy of On-body Sensing in Motion Capture Application
}

\author{
Roya Haratian ${ }^{1}$, TijanaTimotijevic ${ }^{2}$, and Chris Phillips ${ }^{3}$ \\ School of Electronic Engineering and Computer Science \\ Queen Mary University of London \\ ${ }^{1}$ r.haratian@qmul.ac.uk, ${ }^{2}$ t.timotijevic@qmul.ac.uk, ${ }^{3}$ chris.i.phillips@qmul.ac.uk
}

\begin{abstract}
Motion capture coupled with on-body sensing and biofeedback are key enabling technologies for assisted motor rehabilitation. However, wearability, power efficiency and measurement repeatability remain the principle challenges that need to be addressed before widespread adoption of such systems becomes possible. The weight and the size of the on-body sensing system needs to be kept small, and the system should not interfere with the user's movements or actions, but in general they are bulky due to their power consumption requirements. Furthermore, on-body sensors are very sensitive to positioning, which causes increased variability in the motion data. Isolating the characteristic patterns that represent the most important motion data affected by random positioning errors, while also reducing the power consumption, is our main concern. We consider an automated computational approach to address the two problems. We investigate the use of f-PCA for signal separation, whilst accounting for variability in the sensor position. In the designed experiments, we use human subjects and a robot arm to generate motion data, which is analogous to the human joint flexion-extension motion. The data are captured by an active marker-based motion capture system. As both the motion capture system and the robot arm are very accurate in their operation, we are able to introduce deliberate placement errors in a precisely controlled manner. The results are independent from the technology used to measure motion because we consider joint angles as variables in our analysis. The proposed approach can thus be applied to other motion capture systems. The proposed post-processing technique can compensate for uncertainties due to sensor positional changes, whilst allowing greater energy efficiency of the sensors, thus enabling improved flexibility and usability of on-body sensing.
\end{abstract}

Keywords: Motion capture, on-body sensing, sensor power efficiency, sensor placement. 


\section{Introduction}

Wearable computing devices are used to measure human body parameters via on-body sensors. These systems can capture different kinds of information such as motion and physiological activities [1]-[2]-[3]. Our work is motivated by the foreseen need for motion capture applications of home-based motor learning, for example, for assisted rehabilitation or ongoing motor training of disabled users, where wearable system design needs to consider the wearers' comfort and fitting requirements whilst targeting measurement performance. The weight and size of the system needs to be small, and the system should not interfere with the user's movements or actions, especially in motion capture applications [4]. Motion can be captured by placing sensors directly on the body, but they can be cumbersome, uncomfortable to wear and potentially easy to displace. These systems are sensitive to, and highly dependent on, changes in position of sensors, which affect measurement accuracy. This could seriously undermine the effectiveness of self-administered motor training.

In order for motion capture system to be widely adopted as an assistive technology for home-based user-driven rehabilitation, they need to be robust to user placement errors, as well as portable and energy efficient [5]. In motion capture, failure to place markers/sensors accurately is probably the single greatest contributor to measurement variability [6], which is a key impediment to the wider adoption of home-based assistive rehabilitation. Placing markers accurately with respect to specific anatomical landmarks and determining the location of joint centers and other anatomical features in relation to these markers are very important in determining the anthropometry of an individual subject to derive motion data [7]-[8].

This problem of accurate placement is likely to be exacerbated if the sensors or markers are to be positioned by non-expert users. Existing systems require tight fitting of the garment for precise sensor positioning [9]-[10]. Although close textile-body coupling is an advantage in sensor applications, it is uncomfortable and therefore not conducive to committed/persistent motor training that is necessary in long-term rehabilitation or motor function treatments. For looser garments, selecting appropriate sensor positions correctly is essential and is therefore carried out by experts. This prevents a wider adoption of such systems into user populations that would hugely benefit from them, such as rehabilitation patients requiring long-term treatment, or patients with disabilities needing on-going motor training to maintain function [11]-[12].

Sensor misplacement adversely affects the reliable measurement of joint angles. Determining anatomical landmarks position to derive joints centers is influenced by sensor placement [13]. There are substantial differences between the ability of experts and self-marking operators to correctly determine anatomical landmark locations [14]. In [15] it has been shown that hip joint center mislocation of $30 \mathrm{~mm}$ in the anterior-posterior direction generates a mean error on the flexion/extension moment of about 22\%. Physiological knee motions were observed in [16] such as the "screw-home" mechanism even when such motion did not occur, which was due to landmark position uncertainties. These uncertainties can lead to erroneous clinical interpretation of the 
estimation and thus misdiagnosis in rehabilitation.

Body sensing platforms for monitoring of various biological and physiological signals face the challenge of achieving lowpower consumption as well. The overall size of the electronic part of wearable systems is generally dominated by the size of the batteries. Hence to have less bulky systems, sensors need to operate with low power consumption. Changing the sampling rate has been used as a technique for reducing the power consumption. Adaptive sampling is established as a practical method of reducing the sample data volume and thus increasing the energy efficiency [17]. In [18] a low-power analogue system is proposed, which adjusts the converter clock rate. The system allows a low-power and small-sized implementation that can ultimately be integrated into a body-area network sensor. Reducing sampling rate by compressive sampling approaches is a novel sensing/sampling paradigm asserting that one can recover certain signals from far fewer samples or measurements than traditional methods using Nyquist rate [19].

Context-aware sensing known as episodic sampling for wearable sensor technologies has been introduced in [20]. The technique requires a trade-off between energy reduction and accuracy of the system. The effect of reducing the sampling rate of a wearable posture recognition system has been studied in [21]. It is shown that the performance of the posture classification is insensitive to reductions in the sampling rate from $100 \mathrm{~Hz}$ down to $10 \mathrm{~Hz}$ for inertial sensors.

Our work shows how statistical signal processing techniques can be used to compensate for uncertainties in motion data due to random placement errors, while also reducing power consumption. We use functional data analysis techniques, including fitting functions on data sampled at a reduced rate, and principal component analysis to overcome undesirable signal variability. The motivation in applying functional data analysis on human motion data are firstly, smoothness of motion data; and secondly, the principle that the best unit of information is the entire observed function rather than a string of numbers. It is assumed that human motion data have an underlying functional relationship governing them [22]-[23].

Removal of undesirable signals from a superposition of source signals is one of the challenges in signal processing. Filtering strategies for suppression of unwanted source signals have traditionally involved a linear decomposition of signals. The approach is to re-express the original data along a new coordinate system such that the signal of interest can be separated from other sources in the original data while projecting along different bases. By keeping the bases describing the signal of interest and rejecting the rest, filtering can be achieved. Depending on the means by which the bases are determined, these methods can be classified into a priori and adaptive categories.

In a priori approaches the bases are defined independently of the data, like frequency based Finite Impulse Response (FIR) or Infinite Impulse Response (IIR) filters. Discrete Fourier Transform (DFT) filtering has been widely used in clutter rejection [24][25]. Although DFT-based methods have been widely used, they underperform when the frequency characteristics of the unwanted signal and the signal of interest overlap. To overcome this challenge adaptive frameworks for determining basis 
functions have been introduced. In adaptive approaches the bases are determined adaptively from the data. ICA (Independent Component Analysis), PCA (Principal Component Analysis) and SVF (Singular Value Filter) based signal separation methods are examples of adaptive filtering. In these techniques the basis functions are determined adaptively using statistical properties of the data.

ICA based filtering techniques separate unwanted sources of variation from the desired signal by maximizing the entropy of the data transformed linearly into the ICA component axes [26]-[27]. In PCA, the basis functions are determined adaptively from the covariance properties of the data [28]. PCA finds orthogonal directions of greatest variance in the data, whereas ICA component maps may be non-orthogonal. SVF differs from PCA based approaches by incorporating a weighting function that computes non-binary filter coefficients adaptively from information contained in the singular value spectrum [29].

In this paper we show the superiority of functional PCA (f-PCA) over PCA and other introduced filtering approaches with respect to compensation of motion data variance, even when the sampling rate is reduced. To our knowledge, this technique has hitherto not been employed in the context of reducing undesirable motion capture variability and improving power efficiency. The proposed signal processing approach is used as a post-processing technique. It can be used in motion capture laboratories for filtering the data and then returning the "cleaned" information from which undesirable variations have been removed, to clinicians for assessment or to automated motion analysis and biofeedback subsystems of an assisted rehabilitation system. Our method is described in Section II, where we explain the motion data acquisition process and experiment design. The signal processing techniques applied are introduced in Section III. The experimental results, which validate the applicability of the described signal processing techniques, are given in Section IV. Section V concludes the paper.

\section{Method}

In our experiments we use a marker based motion capture system which is considered the "gold standard" in this field due to the measurement precision [8]. Human and Robot arm motion, which is analogous to flexion and extension of human joint, are captured. The robot arm movement is repetitive with high accuracy which can simulate human joints motion in repetitive movements. We used this equipment because its high precision limits sources of variation except for the ones that we intentionally invoke. We consider joint angles as motion data in our analysis. This has the benefit of making the results independent from the technology used to measure motion allowing the proposed approach to be generalized for use with other motion capture technologies.

\section{A. Motion Data Acquisition Process}

The motion capture system used in the study has been described in detail in [8]. Here we outline it only briefly. We use a general-purpose 3D commercial optical motion capture system named Codamotion. The measurement unit contains three prealigned solid-state cameras which track the position of active infra-red LED markers, in real-time. The markers are powered 
from small battery boxes (Fig. 1), which attach to the subject at specific landmarks via solid incompressible wands and plates designed for specific body segments.

A robotic arm [30] was used to measure the effect of random marker placement errors during controlled rotations of one rigid body segment with respect to another (Fig. 2). The robot has a movement positional repeatability of $\pm 0.02 \mathrm{~mm}$. When defining rigid body segments for three dimensional kinematic analysis, a minimum of three markers are required per segment: two markers define the longitudinal axis of the segment (in our laboratory, we define the longitudinal axis as the $\mathrm{Z}$ axis; anteriorposterior axis is the $\mathrm{X}$ axis and the medio-lateral axis is the $\mathrm{Y}$ axis), and the third marker is placed off center from the $\mathrm{Z}$ axis to define the segment as a rigid body in motion capture model [31]-[32].

\section{B. Experiment Design}

Referring to our previous published work, [7], we used the same setting for experiment design on human subject. In addition, we designed the robot arm experiments and our concern is energy efficiency of the system while compensating for variability in the motion data due to random changes in the position of the sensors. We used a robot arm due to its ability to repeat actions with high precision. The robot arm was modeled as two rigid bodies; the upper segment defined as rigid body 1 and the lower defined as rigid body 2 . The robot arm motion of 130 degrees rotation was recorded during intervals of fifteen seconds. Each marker was moved in a random direction by a distance of $2 \mathrm{~cm}$ from the initial reference position in the plane of the body segment. We used a random number generator to derive the random position of sensors within a radius of $2 \mathrm{~cm}$ from the center of each joint. The random number generator generates a random angle between 0 and 360 degrees. It also provides a random radius between 0 and $2 \mathrm{~cm}$.

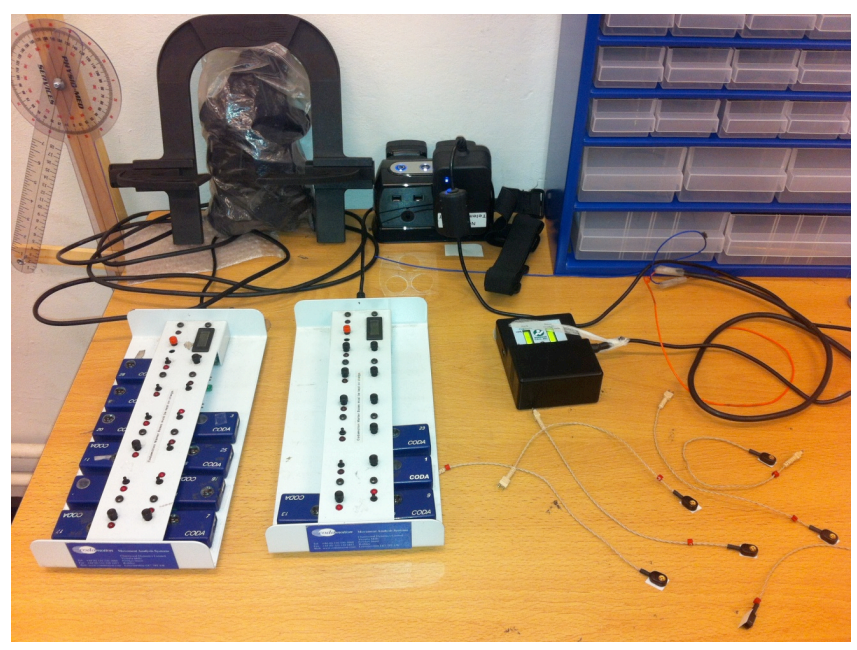

Fig. 1. Markers and drive boxes of the on-body sensing system 


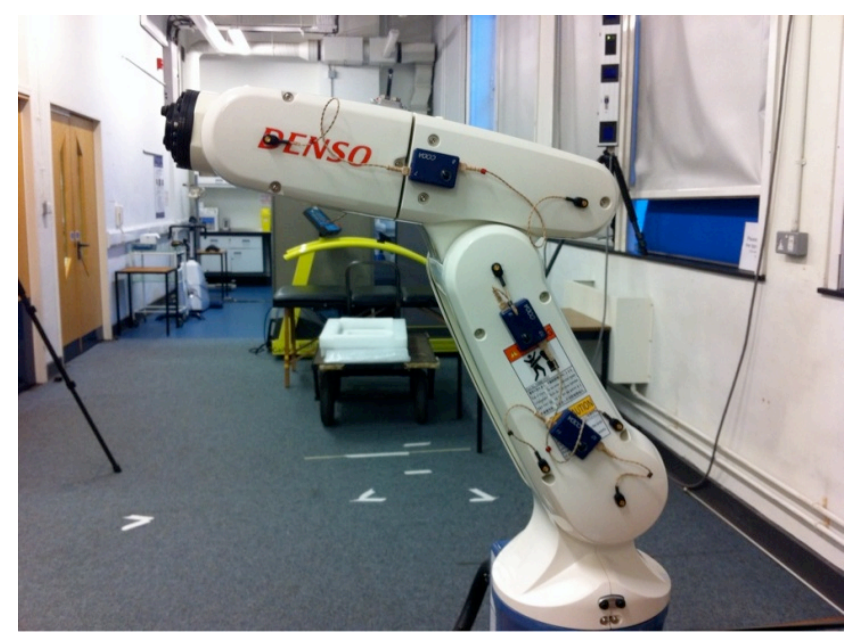

Fig. 2. The configuration of markers on the robot arm that was used on both sides of each segment. The large black boxes contain the batteries and electronics.

In the first step of the robot arm experiment, we gradually reduced the sampling rate to see whether or not the energy efficiency of the on-body sensors will increase. We collected data from several motion capture sessions, each lasting 15 seconds. The voltage level of sensor batteries was measured before and after each session. The chosen sampling rates were 200, 100, 50, $25,10,5,2,1 \mathrm{~Hz}$. The measured difference between voltage level of sensors before and after motion capture at different frequencies is due to the change in the sampling frequency.

In the next step of the robot arm experiment, we carried out ten sessions of marker wearing on the robot arm to explore the combined effect of lowered sampling rate and reduced accuracy of motion capture due to random changes in positions of sensors. We performed 10 motion capture sessions for each of 8 different sampling frequencies $(200,100,50,25,10,5,2,1$ $\mathrm{Hz}$ ), with sensors in randomly changed positions. That is, one randomization of sensor positions was captured for 8 different frequencies, to enable comparisons and analysis of statistical significance. Therefore, we have the robot arm motion data for each frequency 10 times, each time with different random changes in sensors' positions. The captured motion data of different marker wearing sessions differ in the number of samples per session, and in the variation due to random changes in position of sensors.

\section{The Signal Processing Technique}

We use PCA and f-PCA as source separation techniques to see if the variations due to reduction in sampling rate and random changes in position of sensors can be compensated for simultaneously. In this section we briefly introduce PCA and f-PCA as filtering techniques for separation of deterministic and stochastic components in the signal.

\section{A. Principal Component Analysis}

Principal component analysis is a technique in statistical data analysis and feature extraction. For a brief mathematical description of the signal processing technique, we consider the multivariate motion data in the form of $N$ different real-valued, 
time dependent motion vectors of each joint corresponding to $N$ motion capture sessions [33]. Each motion vector consists of $M$ time samples. So there would be $N$ motion vectors, one for each session, each comprising $M$ time samples. We combine these $M$ dimensional vectors into a single $M$-by- $N$ motion matrix per joint angle as it is shown in (1). For $L$ joint angle motion signals, we have $L$ such matrices (in our case, $L=15$ ).

$$
\mathbf{X}=\left[\begin{array}{ccc}
x_{1}[1] & \cdots & x_{N}[1] \\
\vdots & \ddots & \vdots \\
x_{1}[M] & \cdots & x_{N}[M]
\end{array}\right]
$$

Data need to be standardized, so they will have zero mean and unit variance, forming the matrix $\mathbf{Y}$ as shown in (2). The standardization affects the PCA results; the covariance matrix of standardized data is equal to the correlation coefficient matrix and the eigenvectors are normalized to unity. The $j$ th columns of $\mathbf{Y}$ form $M$-dimensional vectors, which are the standardized time sequences of the $j$ th column of $\mathbf{X}$.

$$
\mathbf{Y}=\left[\begin{array}{ccc}
y_{1}[1] & \cdots & y_{N}[1] \\
\vdots & \ddots & \vdots \\
y_{1}[M] & \cdots & y_{N}[M]
\end{array}\right]
$$

Linear combinations of the original variables with maximum variance result in achieving principal components (PCs). The PCs are obtained from the $N$ eigenvalues $\lambda_{\mathrm{i}}$ and eigenvectors $\overrightarrow{e_{l}}$ of data covariance matrix, Cov $\mathrm{ij}_{\mathrm{j}}$, which is defined as $\operatorname{Cov}_{i j}=\left\langle\left[y_{i}(t)-\left\langle y_{i}(t)\right\rangle_{T}\right]\left[y_{j}(t)-\left\langle y_{j}(t)\right\rangle_{T}\right]\right\rangle_{T}$. Hence data can be represented as (3), where $\xi_{\mathrm{n}}(\mathrm{t})$ are the corresponding time series called "scores". The number of principal components is equal to the number of original signal vectors, $N$. Principal components also referred to modes and are shown by $\vec{e}_{i}^{(n)}$.

$$
\bar{y}_{l}=\sum_{n=1}^{N} \xi_{n}(t) e^{(n)}
$$

Principal components correspond to the eigenvectors directly. Each eigenvalue represents a measure of the variance, deviation or spread of the data along the corresponding mode or principal component. Usually, the eigenvalues are ordered from the highest to the lowest: $\lambda_{1}>\lambda_{2} \ldots \lambda_{\mathrm{N}-1}>\lambda_{\mathrm{N}}$. The total population variance is given by the sum of all eigenvalues: Total Variance $=\lambda_{1}+\lambda_{2}+\cdots+\lambda_{N-1}+\lambda_{N}$. The percentage of the total variance in the data explained by the $\mathrm{i}^{\text {th }} \mathrm{PC}$ is given by:

$$
\text { Explained variance }(i)=\frac{\lambda_{i}}{\lambda_{1}+\lambda_{2}+\cdots+\lambda_{N-1}+\lambda_{N}}
$$

Thus, filtering of data that preserves the significant variance within the data but eliminates small variance contributions can be achieved by inverse transformation from the subset of PCs that contain most of the data variance, e.g. from the first $P$ PCs, where 
$P<N$. This feature is used to remove the variance due to random placement errors, as these contribute relatively smaller proportion of variance to the data when compared to the inherent variability of the motion data.

\section{B. Functional Principal Component Analysis}

A useful technique to separate deterministic and stochastic components of movement patterns while preserving the timedependence of the signals is f-PCA. It allows for separation of the main and residual components of a data set. Considering consistent features as coherent components implies the mechanisms generating these common structures follow deterministic rules, otherwise they would not be consistent. In contrast, the residual components often contain a degree of randomness or stochasticity.

This technique is an extension of the PCA statistical technique, where the principal components are represented by functions rather than vectors [34]. The upper limit number of principal components in the multivariate case is the number of variables, while in f-PCA the number of eigenfunctions is equal to a minimum of $(K, N)$, where $K$ is the number of basis functions, and $N$ is the number of variables [22]. The number of basis functions $K$ should be less than or equal to the number of sampled data points, M. An exact representation is achieved when $K=M$. The smaller $K$ is and the better the basis functions reflect certain characteristics of the data, the less computation is required. However, if we make $K$ too small, we may miss some important aspects of the function that we are trying to estimate. A larger $K$ provides a better fit to the data. By considering the mentioned challenges, we selected the number of basis functions equal to the number of variables which is equal to 10 .

In the first step, we should fit function to the data for each motion vector. To fit a function to our data, we use a set of functional building blocks $\emptyset_{k}, k=1,2, \ldots, K$, called basis functions which are combined linearly (5). That is, a function $x(t)$ fitted to the measured data is expressed as a basis function expansion;

$$
x(t)=\sum_{k=1}^{K} c_{k} \emptyset_{k}
$$

Parameters $c_{k}$ are the coefficients of the expansion. The matrix expression of $N$ functions can be expressed as $\mathrm{X}(\mathrm{t})=\mathrm{C} \emptyset(\mathrm{t})$, where $\mathrm{X}(\mathrm{t})$ is a vector of length $N$ containing the functions $\mathrm{x}_{\mathrm{i}}(t)$, and the coefficient matrix $\mathrm{C}$ has $N$ rows and $K$ columns. The functional eigenequation is

$$
\int v(s, t) \xi(t) d t=\rho \xi(s)
$$

where $\rho$ is an eigenvalue and $\xi(s)$ is an eigenfunction of the variance-covariance function. The eigenfunction which is called principal component weight function, $\xi_{1}(\mathrm{~s})$, can be found by (7): 


$$
\text { Subject to } \int \xi_{1}^{2}(s) d s=\left\|\xi_{1}\right\|^{2}=1,
$$

where the principal component score is defined as:

$$
f_{i 1}=\int \xi_{1}(s) x_{i}(s) \mathrm{d} s
$$

Eigenvalues can be constructed stepwise by requiring each new eigenfunction computed in step 1, to be orthogonal to those computed in the previous steps,

$$
\begin{gathered}
\int \xi_{j}(t) \xi_{l}(t) d t=0, \quad j=1, \ldots, l-1 \\
\int \xi_{l}^{2}(t) d t=1 .
\end{gathered}
$$

Deterministic and stochastic components of movement patterns can be separated by using f-PCA as a filtering technique, particularly when partitioning signals into deterministic and stochastic components. This is achieved by subtracting either one or other from the signal and can be regarded as filtering the noise or the common parts, respectively. Random changes in positions of sensors causes a random effect on the motion data, therefore to separate this effect from the main and coherent component of

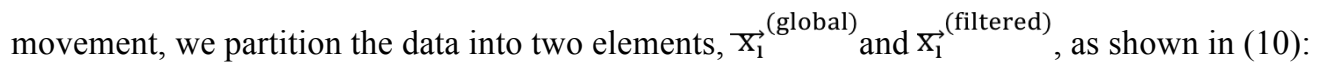

$$
\begin{aligned}
& x_{\imath}={x_{l}}^{(\text {global })}+x_{l}^{(\text {filtered })} \\
&=\sum_{\mathrm{n}=1}^{\mathrm{L}<\mathrm{N}} \xi_{\mathrm{n}}(\mathrm{t}) \mathrm{f}_{\mathrm{i}}^{(\mathrm{n})}+\sum_{\mathrm{n}=\mathrm{L}+1}^{\mathrm{N}} \xi_{\mathrm{n}}(\mathrm{t}) \mathrm{f}_{\mathrm{i}}^{(\mathrm{n})}
\end{aligned}
$$

Here we assume the number of basis functions is greater than the number of variables. The sum of the dominant principal components weight functions is given by ${\mathrm{x}_{1}}^{\text {(global) }}$, so the filter characteristic depends on the data. The number of modes that define the global pattern influences the filtered pattern. We select the most relevant principal component weight function, the one with largest variation, and remove the rest from the f-PCA domain. We select the PCs that reflect at least 95 percentage of variance. The percentage of the total variance can be calculated by (4). The data are then returned to the original signal domain, but without the eigenfunctions containing smaller variations. This gives us filtered, cleaner data where random variation due to placement errors is removed. 


\section{Results and Discussion}

The mean variance before and after applying the techniques reviewed in the Introduction section has been calculated for each of the subjects' body angles in 10 marker sessions as explained in [7] to compare the performance of the proposed approach with other techniques. The percentage of mean variance improvement in the $\mathrm{X}, \mathrm{Y}$, and $\mathrm{Z}$ direction after applying different techniques is shown in Fig. 3, 4 and 5. The values in these figures are for normalized data. The angles are pelvis, hip, knee, ankle and foot. Results of applying fast DFT (FFT), SVF, ICA and PCA based filtering techniques are compared with the proposed fPCA one. We use two algorithms for Independent Component Analysis based on Maximum Likelihood (ICA ML) and ICA Molgedy and Schuster (MS) [35].

We considered the improvement as a percentage of variance change in the data before and after applying the filtering techniques. Results show that as random changes in the position of sensors are introduced, variation in motion data increases. We can see that the performance of fPCA and PCA are similar. Other techniques perform worse, especially FFT-based filtering as it just removes the high frequency patterns in the data, and thus is unable to separate stochastic patterns from the deterministic one. The ICA based filtering technique causes more ripple in the data so it cannot separate the dominant mean of variation in the derived angle motion data. This is because ICA based signal separation performs properly on non-Gaussian distributed data. The SVF-based filter performs worse than fPCA since the dominant mode of variance in the data in PPCA-based filtering is reflected in the first few principal components.

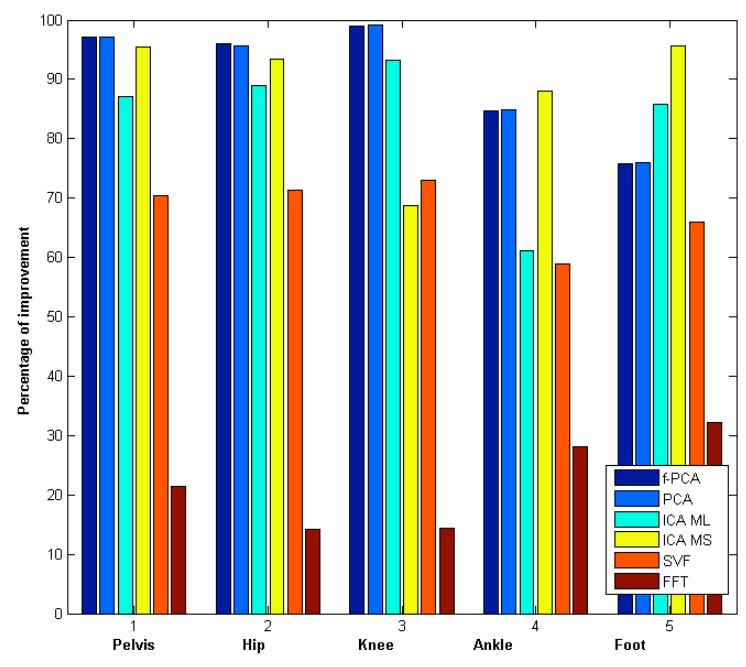

Fig. 3, Mean percentage of variance improvement in the $\mathrm{X}$ direction. 


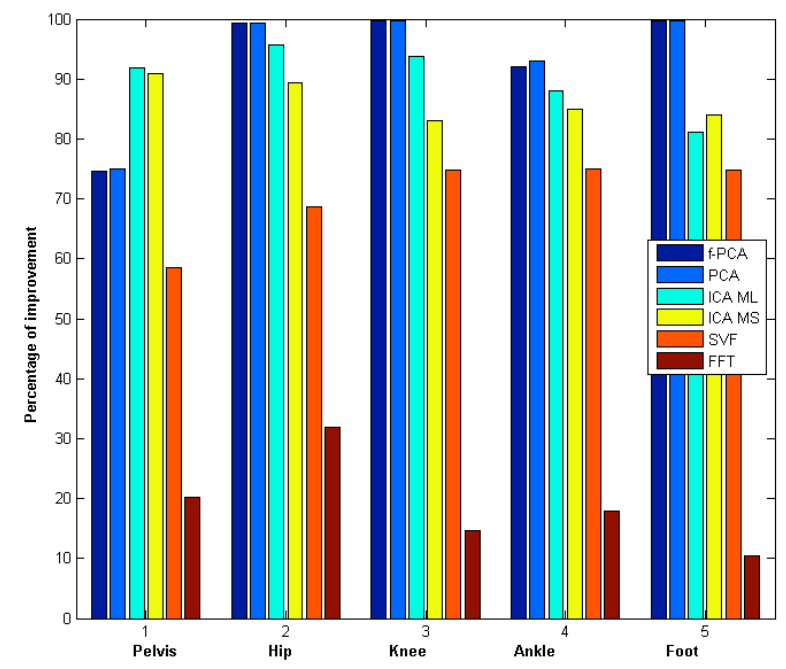

Fig. 4, Mean percentage of variance improvement in the $\mathrm{Y}$ direction.

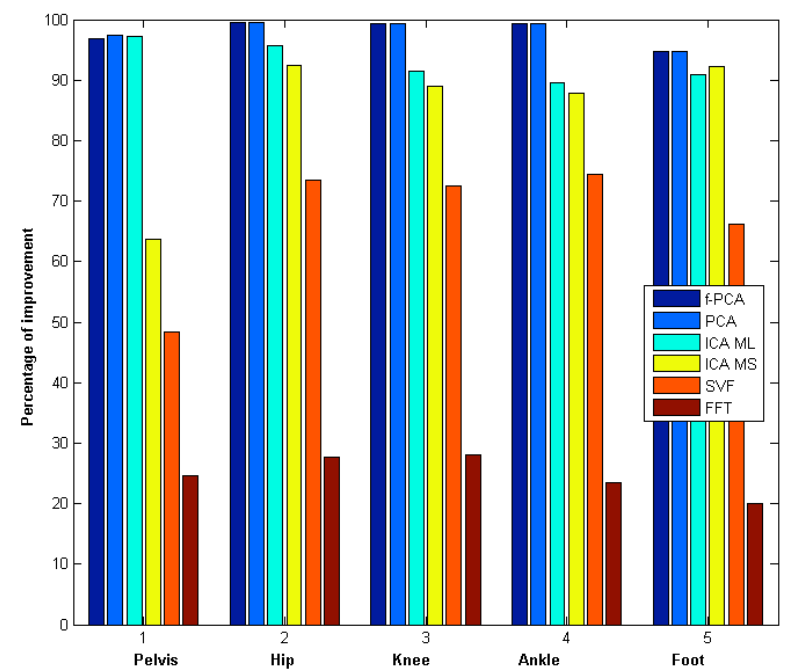

Fig. 5, Mean percentage of variance improvement in the $\mathrm{Z}$ direction.

In the robot arm experiments, the voltage before and after motion capture session in different frequencies was measured in 15 seconds intervals for the six sensor boxes. We measured all sensor voltages in the same, full condition of sensor battery. Fig. 6 shows that as sampling frequency rate increases, voltage drop within the fixed time of the measurement session, increases as well. It shows the impact of reduction in frequency on energy usage. There is a reduction in power consumption due to decreasing sampling rate. 


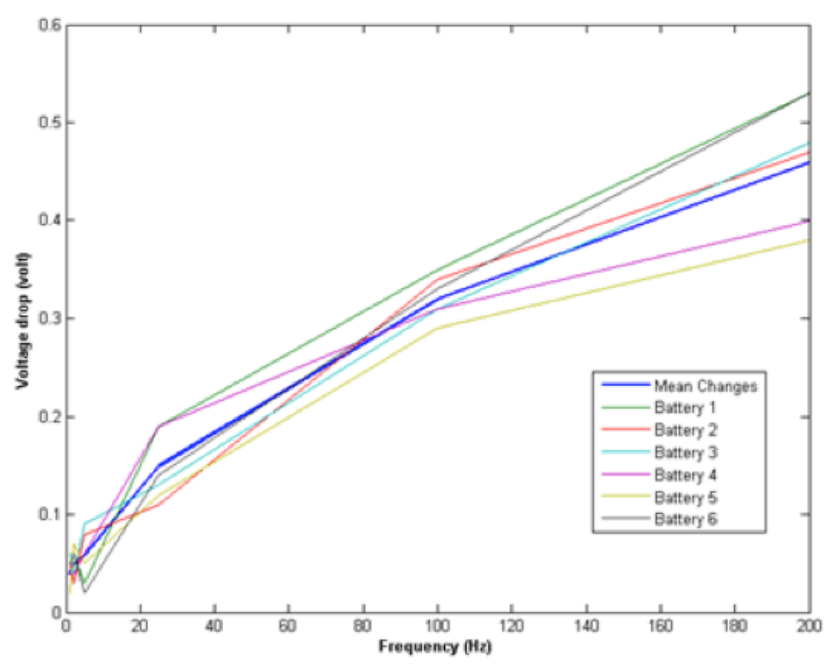

Fig. 6. Voltage drops in sensors batteries versus frequency of sampling.

Regarding the next step of the robot arm experiment, data variance of ten marker wearing sessions for each sampling frequency was calculated as the square of the standard deviation of each joint angle motion signal. The variances of unprocessed and processed captured motion data sampled at different frequency rates are shown in Table 1. As expected, the reduction in sampling rate increases the variance. Results show that down to the Nyquist frequency the variance is of the same order of magnitude. Although there is an increase in magnitude of variance from $100 \mathrm{~Hz}$ to $200 \mathrm{~Hz}$, this is an insignificant amount that can be due to high frequency noise. The residual high frequency noise in the position measurement arises mainly from photodetector current noise in the cameras and the effects caused by room lighting [8]. In motion capture, these kinds of noise are usually compensated for by using a low pass filter. A further reduction in sampling rate below the Nyquist frequency causes a significant increase in the variance. However, applying the filtering technique compensates for the increase in the variance. The results show that f-PCA outperforms PCA in terms of data variance, as illustrated in Fig. 7.

Table 1

Variance change before and after applying PCA and f-PCA

\begin{tabular}{c|l|l|l|l|l}
\hline \multirow{2}{*}{$\begin{array}{c}\text { Frequency } \\
(\mathbf{H z})\end{array}$} & Before & \multicolumn{2}{|c|}{ After } & \multicolumn{2}{l}{ Percentage of change (\%) } \\
\cline { 3 - 6 } & & PCA & f-PCA & PCA & f-PCA \\
\hline $\mathbf{2 0 0}$ & 7.6601 & 6.9826 & 4.8996 & 8.84 & 36.04 \\
\hline $\mathbf{1 0 0}$ & 6.7113 & 6.1446 & 4.1501 & 8.44 & 38.16 \\
\hline $\mathbf{5 0}$ & 6.1156 & 5.5804 & 3.6543 & 8.75 & 40.25 \\
\hline $\mathbf{2 5}$ & 6.0807 & 5.0608 & 3.2224 & 16.77 & 47.01 \\
\hline $\mathbf{1 0}$ & 10.8290 & 4.1176 & 2.6794 & 61.98 & 75.26 \\
\hline $\mathbf{5}$ & 16.1705 & 7.8966 & 5.8977 & 51.17 & 63.53 \\
\hline $\mathbf{2}$ & 41.5028 & 6.6151 & 4.7679 & 84.06 & 88.51 \\
\hline $\mathbf{1}$ & 273.5735 & 25.4157 & 17.7664 & 90.71 & 93.51 \\
\hline
\end{tabular}




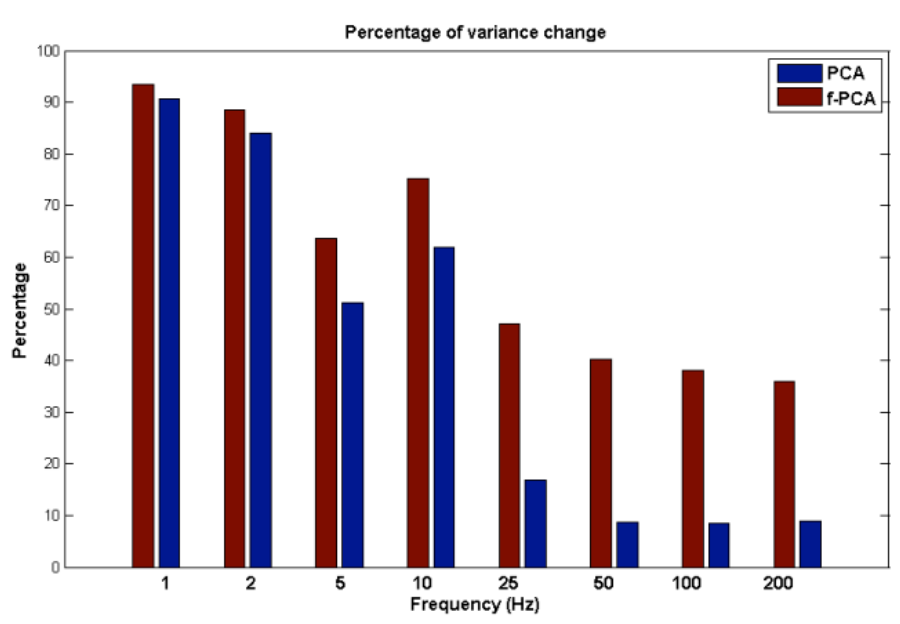

Fig. 7. Percentage of variance change for different frequencies before and after applying PCA and F-PCA based signal separation techniques.

It is shown in Fig. 7 that as the sampling rate reduces, the improvement will increase. But by comparing PCA and f-PCA filtering results we see that f-PCA can better compensate for variations due to random changes in the positions of sensors than PCA. The advantage of f-PCA over PCA is more obvious at higher frequency sampling rates. Figure 7 shows that f-PCA works better than PCA at all frequencies, although we see a change in the trend of relative improvement below $25 \mathrm{~Hz}$, which is due to sampling below the Nyquist frequency. We know that the sampling rate must be at least twice the maximum frequency present in the signal, which is called the Nyquist rate. In the motion data that we captured the Nyquist rate is $20 \mathrm{~Hz}$, which explains the reduction in the relative improvement below $25 \mathrm{~Hz}$.

Depending on the user preference and application goals, the sampling frequency can be selected among the available range of the system frequencies. If the goal is accuracy of the captured data, higher frequencies are recommended. Lower frequencies are suggested in the cases where the goal is lowering the energy usage of sensors, for example, to extend capture time in field applications. So by decreasing sampling rate we can increase life time of sensors' batteries. The life of a battery can be given either as the number of charge and discharge cycles that can be delivered or as the total lifetime in years. Factors which affect the battery life are the operating temperature, the discharge depth, and the charging regime. When the average current drain on the battery is lower, the discharge time or service life to the end of the battery life is longer [36]. The average current $\left(I_{a v g}\right)$ can be calculated by following equation.

$$
I_{\text {avg }}=\frac{t_{0} I_{0}+t_{1} I_{1}+\cdots+t_{n} I_{n}}{t_{0}+t_{1}+\cdots+t_{n}}
$$

In this equation $t_{0}, t_{1}, \ldots, t_{n}$ shows the time intervals that the battery provides current, $I_{0}, I_{1}, \ldots, I_{n}$, for the connected circuit. If the time intervals during which the battery provides current to the circuit are of a shorter duration, the battery can provide power for motion capture sessions over a longer period. It means that when there is a reduction in sampling rate of markers attached to the batteries, the total time during which the current will be needed will decrease. The smaller current requirement will enable longer motion capture intervals between battery recharging. 
Assuming a specific time interval for motion capture in different sampling rates, and equal active time in each period for all frequencies, $t_{i}=t$, the total time that each sensor drain current from batteries is calculated by: $\sum_{i=0}^{K_{i}} t$. In a specific time interval of motion capture, for higher frequencies the number of active time slots, $K_{i}$, is higher. It can be written as $f_{1}<f_{2}<\ldots<f_{m}$, then $K_{1}<K_{2}<\ldots<K_{m}$. Therefore:

$$
\sum_{i=1}^{K_{i}} t \quad<\sum_{j=1}^{K_{j}} t \quad \text { for } \quad K_{i}<K_{j}
$$

which means the total time that the sensors drain current from batteries is greater in higher frequencies. According to equation (11), the service life of the battery, which is dependent on average current drain, will be increased by decreasing the sampling rate so longer system life time.

The proposed signal processing approach adds processing complexity to the motion capture procedure of the order of $2\left(O(n \log n)+O\left(\log \left(n m^{2}\right)\right)+O\left(m^{3}\right)\right)[35]$. In this formula $n$ is number of samples and $m$ is the dimensionality which is equal to the number of variables. However, since on-body sensing systems usually have a central system for analyzing the data that is located far from the body and connected to a powerful processing unit with a connection to an abundant electricity supply, processing complexity is not a challenge in this case.

To test for statistical significance of our results, we carried out a multiple comparison test on the data. In this test we considered variance in the data as the dependent variable, and the sampling frequency and applied techniques as independent variables. We used a two-way ANOVA (Analysis of Variance) test on the results produced at different sampling frequencies. For each test, data were grouped into 4 categories. The first group is the baseline where there is no frequency change (i.e. the sampling rate is the standard $200 \mathrm{~Hz}$ ), and the filtering techniques are not applied on the data. The rest of the results were grouped into three treatment groups, depending on whether either or both of the two independent treatments, that is, reducing the sampling rate and/or application of the f-PCA technique, where applied.

The hypothesis is that there is significant difference in variance after applying the proposed approach from the baseline where the filtering technique was not applied on the data sampled at the $200 \mathrm{~Hz}$ standard sampling rate. The test results show that there is a sufficiently small $p$ value (less than 0.05 ) for the hypothesis. It means there is a significant difference from the baseline and the marginal means of the 3 groups' populations in the test. This implies that there is a substantial effect due to the application of the proposed technique. The multiple comparison test confirms that reducing the frequency increases the variance in the data significantly and applying the f-PCA technique compensates for this increase in the variance. Taking all these results together, it is clear that PCA-based signal separation techniques can be useful in removing unwanted variation in motion data and enable a more flexible assisted rehabilitation system for sensor-based motion capture. 


\section{Conclusion}

In this paper we studied technical challenges related to portability of on-body sensing systems specifically with regard to motion capture applications. We considered power consumption and positional uncertainties as two main issues in these systems. By proposing a post-processing approach we showed how these challenges can be overcome. The motion of human subjects and a robot arm were captured whilst "random" changes were made to the positions of sensors for different sampling frequencies. The movement of robot arm was analogous to flexion and extension of a human body joint. Ten marker wearing sessions of the robot arm were captured for each different sampling frequency whilst introducing random changes in the positions of the sensors. Results show that despite the inherent accuracy of both the motion capture system and the repetitive robot arm movements, significant variation in the motion data exists due to these random changes in the positions of sensors as well as variation in the sampling frequency. Reducing the sampling rate has the benefit of lowering the power consumption and therefore permits the use of smaller batteries leading to improved comfort for the wearer. However, as the sampling frequency decreases, the variation in patterns of motion increases. Nevertheless, we have shown that after applying f-PCA and PCA signal processing techniques the increased variation due to sampling frequency reduction, as well as the variations due to errors in marker positioning can be substantially reduced, thus providing a step towards a robust and flexible assisted rehabilitation. Furthermore, we considered joint angles as motion data in our analysis. This has the benefit of making the results independent from the technology used to measure motion, and allows the proposed approach to be generalized for use with other motion capture technologies.

\section{References}

1 Pantelopoulos, A., Bourbakis, N.: 'A survey on wearable sensor-based systems for health monitoring and prognosis', IEEE Transactions on Systems, Man, and Cybernetics, , 2010, 40, (1), pp 1-12

2 Bonato, P.: 'Wearable sensors/systems and their impact on biomedical engineering', IEEE Engineering in Medicine and Biology Magazine, 2003, pp 1820

3 Hanson, M. A., Powell Jr, H., Barth, A. T.: 'Body area sensor networks: challenges and opportunities', Computer, January 2009, pp 58-65

4 Tang, S.: 'Recent developments in flexible wearable electronics for monitoring applications', Transactions of the Institute of Measurement and Control, 2007, 29, pp 283-300

5 Jovanov, E., Yang, C., Zhang, Y.: 'Body sensor networks: from theory to emerging applications', IEEE Transactions on Information Technology in Biomedicne, 2009, 13, (6), pp 859-863

6 Baker, R.: 'Gait analysis methods in rehabilitation,' Journal of NeuroEngineering andRehabilitation, 2006

7 Haratian, R., Twycross-Lewis, R., Timotijevic, T., Phillips, C.: 'Towards flexibility in sensor placement for motion capture systems: a signal processing approach', IEEE Sensors Journal, 2013

8 Codamotion user guide, Charnwood Dynamics Ltd, 2005

9 Wade, D., Jong, B.: 'Recent advances in rehabilitation', BMJ , 2000, 320, pp 1385-1388

10 Knight, J., Deen-Williams, D., Arvanitis, T., Baber, C., Sotiriou, S., Anastopoulou, S. Gargalakos, M.: 'Assessing the wearability of wearable computers,' in 10th IEEE International Symposium on Wearable Computers, Montreux, Switzerland, 2006

11 Harms, H., Amft, O., Troster, G.: 'Influence of a loose-fitting sensing garment on posture recognition in rehabilitation', in IEEE Biomedical Circuits and Systems Conference, 2008 , Baltimore, MD

12 Harms, H., Amft, O., Tröster, G.: 'Does loose fitting matter? Predicting sensor performance in smart garments', 7th International Conference on Body Area Networks, 2012, Oslo, Norway

13 Croce, U., Leardini, A., Chiari, L., Cappozzo, A.: 'Human movement analysis using streophotogrammetry part 4: assessment of anatomical landmark mislocation and its effect on joint kinematics', Gait and Posture, 2005, 21, pp 226-237

14 Croce, U., Cappozzo, A., Kerrigan, D.: 'Pelvis and lower limb anatomical landmark calibration precision and its propagation to bone geometry and joint angles', Med Biol Eng Comp, 1999, 37, pp 1029-1034

15 Stagni, R., Leardini, A., Cappozzo, A., Bendetti, M., Cappello, A.: 'Effect of hip joint centre mislocation on gait analysis results', Journal of Biomechanics, 2000, 33, pp 1479-1487

16 Piazza, S., Cavanagh, P.: 'Measurment of the screw-home motion of the knee is sensitive to errors in axis alignment', Journal of Biomechanics, 2000, 33, pp 1029-1034 
17 Hamouda, Y., Phillips, C.: 'Adaptive sampling for energy-efficient collaborative multi-target tracking in wireless sensor networks', IET Wireless Sensor Systems, $2010,1,(1)$, pp 15-25

18 Reger, R. and Taylor, J. T.: 'An adaptive sampling system for sensor nodes in body area networks', IEEE Transactions on Neural Systems and Relabilitation, 2009, pp 183-189

19 Candes, E., Wakin, M.: "An introduction to compressive sampling," IEEE Signal Processing Magazin, 2008, pp 21-33.

20 Au, L., Batalin, M., Stathopoulos, T., Bui, A., Kiase, W.: 'Episodic sampling: towards energy efficient patient monitoring with wearable sensors', in 31st IEEE International Conference of the EMBS, 2009, Miniapolis-USA

21 Rendic, R., Gaura, L., Brusey, J., Kemp, J.: 'Wearable posture recognition systems: factors affecting performance', in International Conference on Biomedical and Health Informatics, 2012, Hong Kong-China,

22 Ormoneit, D., Black, M. J., Hastie, T., Kjellstrom, H.: 'Representing cyclic human motion using functional analysis', Image and Vision Computing, 2005, pp 1264-1276,

23 Ramsy, J., Hooker, G., Graves, S.: Functional data analysis, (New York: Springer, 2005)

24 Bjaerum, S., Trop, H., Kristoffersen, K.: 'Clutter filter design for ultrasound color flow imaging', IEEE Transactions on Ultrasonics Ferroelectrics and Frequency Control, 2002, 49, (2), pp 204-216

25 Kadi, A., Loupas, T.: 'On the performance of regression and step-initialized IIR clutter filters for color doppler systems in diagnostic medical ultrasound', IEEE Transactions on Ultrasonics Ferroelectrics and Frequency Control, 1995, 42, (5), pp 927-937

26 Hyvarinen, A., Oja, E.: 'Independent component analysis: algorithms and applications', Neural Networks, 2000, 13, pp 411-430

27 Hyvarinen, A., Karhunen, J., Oja, E.: 'Independant component analysis', (John Wiley and Sons, USA, 2001)

28 Jolliffe, I.: 'Principal Component Analysis', (Springer, New York, 2002)

29 Mauldin Jr, F., Lin, D., Hossack, J.: 'The singular value filter: a general filter design strategy for PCA-based signal separation in medical ultrasound imaging', IEEE Transctions on medical Imaging, 2011, 30, (11), pp 1951-1964

30 Denso robot, setting-up manual, Denso wave incorporated, 2011

31 Hamill, J., Selby, W.: Three dimensional kinematics in Research Methods in Biomechanics, Human Kinetics, Champaign: IL, 2004

32 Trefethen, L., Bau, D., Numerical Linear Algebra, Philadephia, PA,: Society of Industrial and Applied Mathematics, 1997

33 Haratian, R., Timotijevic, T., Phillips, C.: 'A PCA-based technique for compensating the effect of sensor position changes in motion data', in 6th IEEE I nternational Itelligent Systems (IS) Conference, 2012, Sofia

34 Coffey, N., Harrison, A., Donoghue, O., Hayes, K.: 'Common functional principal components analysis: a new approach to analyzing human movement', Human Movement Science, 2011, 30, pp 1144-1166

35 Haratian, R. 'Towards flexibility in on-body sensing systems: a signal processing approach' PhD Thesis, Queen Mary University of London, 2014

36 Linden, D., Reddy, T.: Handbook of Batteries, (McGraw-Hill, New York, 2001) 